TITLE:

Fluid dynamics analyses of the
intrahepatic portal vein tributaries
using 7-T MRI(Dissertation_全文)

$\operatorname{AUTHOR}(S)$ :

Oshima, Yu

\title{
CITATION:
}

Oshima, Yu. Fluid dynamics analyses of the intrahepatic portal vein tributaries using 7-T MRI. 京都大学, 2021, 博士(医学)

ISSUE DATE:

2021-11-24

URL:

https://doi.org/10.14989/doctor.k23573

RIGHT: 


\title{
ORIGINAL ARTICLE
}

\section{Fluid dynamics analyses of the intrahepatic portal vein tributaries using 7-T MRI}

\author{
Yu Oshima ${ }^{1}$, Satoshi Ogiso ${ }^{1}$, Hirohiko Imai ${ }^{2}$, Masanori Nakamura ${ }^{3}$, Satoshi Wakama ${ }^{1}$, \\ Katsuhiro Tomofuji ${ }^{1}$, Takashi Ito ${ }^{1}$, Ken Fukumitsu ${ }^{1}$, Takamichi Ishii ${ }^{1}$, Tetsuya Matsuda ${ }^{2}$ \& Kojiro Taura ${ }^{1}$ \\ ${ }^{1}$ Division of Hepato-Biliary-Pancreatic Surgery and Transplantation, Department of Surgery, Graduate School of Medicine, Kyoto \\ University, 54 Kawara-cho, Shogoin, Sakyo-ku, Kyoto, 606-8507, ${ }^{2}$ Department of Systems Science, Graduate School of Informatics, \\ Kyoto University, Kyoto, 606-8501, and ${ }^{3}$ Department of Electrical and Mechanical Engineering, Nagoya Institute of Technology, \\ Nagoya, Japan
}

\begin{abstract}
Background: Assessing portal vein (PV) hemodynamics is an essential part of liver disease management/liver surgery, yet the optimal methods of assessing intrahepatic PV flow have not yet been established. This study investigated the usefulness of 7-Tesla MRI with hemodynamic analysis for detecting small flow changes within narrow intrahepatic PV branches.

Methods: Flow data in the main PV was obtained by two methods, two-dimensional cine phase contrast-MRI (2D cine PC-MRI) and three-dimensional non-cine phase contrast-MRI (3D PC-MRI). Hemodynamic parameters, such as flow volume rate, flow velocity, and wall shear stress in intrahepatic PV branches were calculated before and after a meal challenge using 3D PC-MRI and hemodynamic analysis.

Results: The hemodynamic parameters obtained using 3D PC-MRI and 2D cine PC-MRI were similar. All intrahepatic PV branches were clearly depicted in eight planes, and significant changes in flow volume rate were seen in three planes. Average and maximum velocities, cross-sectional area, and wall shear stress were similar between before and after a meal challenge in all planes.

Conclusion: 7-Tesla 3D PC-MRI combined with hemodynamic analysis is a promising tool for assessing intrahepatic PV flow and enables future studies in small animals to investigate PV hemodynamics associated with liver disease/postoperative liver recovery.
\end{abstract}

Received 28 July 2020; accepted 6 April 2021

\section{Correspondence}

Satoshi Ogiso, Division of Hepato-Biliary-Pancreatic surgery and Transplantation, Department of Surgery, Graduate School of Medicine, Kyoto University, 54 Shogo-in Kawahara-cho, Sakyo-ku, Kyoto, 606-8507, Japan. E-mail: ogiso@kuhp.kyoto-u.ac.jp

\section{Introduction}

Portal vein hemodynamic parameters such as flow pressure, flow volume rate, and wall shear stress (WSS) are critical factors in liver disease management and liver surgery, as they are closely associated with liver function and liver damage. The progression of chronic liver disease and liver fibrosis causes portal hypertension, which contributes to the development of ascites and gastrointestinal varices. Surgical interventions such as partial hepatectomy, liver transplantation, and preoperative portal vein embolization can lead to portal vein hyperperfusion, which increases the mechanical stress on PV endothelial cells, triggering dramatic liver hypertrophy. ${ }^{1}$ Excessive endothelial stress, in contrast, is reported to impair postoperative liver regeneration and functional recovery, requiring portal vein modulation strategies to prevent liver insufficiency after hepatectomy and liver transplantation. ${ }^{2-4}$

Although the accurate assessment of portal vein hemodynamics is essential to correctly understanding these values' association with liver disease and liver function, methods of monitoring and analyzing changes in intrahepatic portal vein perfusion have not been well established. To date, Doppler ultrasound (DUS) has been the gold standard modality for assessing PV hemodynamics; yet it yields flow velocity data for a two-dimensional scanning area only, and is often blocked by bowel gas. Magnetic resonance imaging (MRI) is a good alternative to the conventional modalities, as it enables noninvasive anatomic and hemodynamic measurements; MR angiography (MRA) for morphologic data and cine phase contrast (PC) MRI 
for time-resolved flow velocity data. In addition, 7-T (7T) MRI possess higher spatial resolution than current standard of 3-T MRI, and three-dimensional (3D) PC-MRI provides hemodynamic data of three-dimensional PV flow. ${ }^{5}$ in any vessel of interest. $^{6}$ while 2D PC-MRI provides that within a single plane. Hemodynamic analysis, meanwhile, allows us to calculate PV flow parameters such as intravascular streamlines, pressure gradients, and WSS, which is the friction stress between the vascular wall and the blood, calculated from the velocity of blood flow near the vascular wall. ${ }^{7}$ This study explores a new method combining 7T MRI with hemodynamic analysis to obtain accurate and detailed hemodynamic data within the intrahepatic PV branches. This tool will improve our ability to study the important and complex relations between liver and portal vein hemodynamics.

\section{Material and methods}

\section{Animals}

Female Lewis rats $(\mathrm{n}=5$, aged $8-12$ weeks, body weight 180-200g; SLC, Hamamatsu, Japan) were used for PV flow analyses. The rats were maintained on a standard laboratory diet and water ad libitum, and were housed in a temperature- and humidity-controlled environment under a constant 12-h light/ dark cycle.

To evaluate the impacts of fasting and eating on PV flow, MR scans were performed before and after a meal challenge. A first MR scan (pre-meal) was performed after at least $16 \mathrm{~h}$ of fasting (water was given). After the first scan, rats were given free access to standard food for $2 \mathrm{~h}$, after which a second MR scan (postmeal) was performed.

During MRI, each rat was placed in a cradle in the prone position. The rat's head was fixed using ear bars and a tooth bar. The animal's electrocardiograph (ECG), respiratory rate, and rectal temperature were continuously monitored using a subdermal needle electrode, pressure-sensitive respiration sensor, and Thermistor temperature probe, respectively (MR-Compatible Small Animal Monitoring \& Gating System, Model 1025, SA Instruments, Inc., Stony Brook, NY, USA, with PC-SAM software V.5.12, SA Instruments). Body temperature was maintained by a flow of warm air supplied into the magnet bore using a heater system (MR-Compatible Small Animal Heating System, SA Instruments). Anesthesia was maintained by means of inhalation of $2-3 \%$ isoflurane in air at $1.4 \mathrm{~L} / \mathrm{min}$ through a face mask. Animals' heart rates ranged from 360 to 430 beats/min, body temperatures ranged from 34 to $37^{\circ} \mathrm{C}$, and respiration rates ranged from 40 to 80 breaths/min.

After the post-meal MRI scan, the animals were sacrificed humanely to retrieve the whole liver, and the weight of each lobe was measured after excess fluid and surrounding tissues had been removed.

All animal experiments were approved by the Kyoto University Animal Experimentation Committee and were performed in accordance with Kyoto University Animal Protection Guidelines.

\section{MRI acquisition}

MR measurements were conducted on a 7-T preclinical scanner (BioSpec 70/20 USR; Bruker BioSpin MRI GmbH, Ettlingen, Germany). A quadrature transmit-receive volume coil (inner diameter $72 \mathrm{~mm}$, T9562; Bruker BioSpin) was used for MR signal detection. MRI data was acquired with a dedicated operation software package (ParaVision 5.1; Bruker BioSpin).

In order to obtain morphological data on the PV tree, respiratory-gated three-dimensional time-of-flight MRA was performed with the following acquisition parameters: pulse sequence, flow-compensated gradient echo sequence; repetition time (TR), $30 \mathrm{~ms}$; echo time (TE), $2.2 \mathrm{~ms}$; flip angle, $25^{\circ}$; field of view (FOV), $32 \times 32 \times 16 \mathrm{~mm}^{3}$; acquisition matrix size, $100 \times 100 \times 50$; isotropic spatial resolution, $320 \mu \mathrm{m}$; axial orientation; number of averages, 2 ; and scan time, $\sim 5 \mathrm{~min}$ (depending on respiration rate).

A respiratory- and cardiac-gated two-dimensional cine phase contrast MRI (2D cine PC-MRI), ${ }^{8}$ was used to acquire data on the flow in the main PV. The image plane of the 2D cine PC-MRI was placed perpendicular to the direction of blood flow with reference to the MRA images. The imaging parameters of the $2 \mathrm{D}$ cine PC-MRI were as follows: pulse sequence, flow-compensated gradient echo sequence with bipolar gradient pulses; TR, $10.5 \mathrm{~ms}$; TE, $2.7 \mathrm{~ms}$; flip angle, $30^{\circ}$; FOV, $32 \times 32 \mathrm{~mm}^{2}$; acquisition matrix size, $160 \times 160$; in-plane spatial resolution, $200 \times 200 \mu \mathrm{m}^{2}$; slice thickness, $2 \mathrm{~mm}$; number of slices, 1 ; number of averages, 8 ; and scan time, $\sim 5.5 \mathrm{~min}$ (depending on heart and respiration rates). The velocity encoding direction was set to the slice direction. The velocity encoding (VENC) values were appropriately adjusted for each scan, i.e., for each individual rat and time point, in the range of $35-40 \mathrm{~cm} / \mathrm{s}$ based on flow velocity. The number of frames was appropriately adjusted for each scan in the range of 12-14 frames/cardiac cycle based on each animal's heart rate.

A respiratory-gated and non-cardiac-gated three-dimensional phase contrast MRI (3D PC-MRI) was used to obtain data on the flow in the whole PV tree, with the following parameters: pulse sequence, flow-compensated gradient echo sequence with bipolar gradient pulses; TR, $30 \mathrm{~ms}$; TE, $2.7 \mathrm{~ms}$; flip angle, 25 ; FOV, $32 \times 32 \times 16 \mathrm{~mm}^{3}$; acquisition matrix size, $100 \times 100 \times 50$; isotropic spatial resolution, $320 \mu \mathrm{m}$; axial orientation; number of averages, 2; and scan time, $\sim 20$ min (depending on respiratory rate). Four velocity encoding gradient steps comprised of combinations of three orthogonal gradients (Hadamard scheme) were used to derive flow velocities for the three orthogonal spatial directions. The VENC values were appropriately adjusted for each scan, i.e., for each individual rat and time point, in the range of $25-40 \mathrm{~cm} / \mathrm{s}$.

Hemodynamic parameters such as flow volume rate, maximum and average flow velocities, vessel cross-sectional area, 
and WSS were calculated for arbitrary regions using iTFlow fluid simulation software (Cardio Flow Design Inc., Tokyo, Japan).

\section{Statistical analysis}

The hemodynamic parameters are expressed as median \pm interquartile range (IQR). Hemodynamic parameters of the intrahepatic PV branches were compared before and after the meal challenge using paired Student t-tests. Parameters obtained by 3D PC-MRI and those obtained by $2 \mathrm{D}$ cine PC-MRI were compared using paired Student t-tests. Parameters by the two methods were also compared using Bland and Altman analysis by calculating the mean $(d)$ and SD of the difference. From these data, the limits of agreement $(d-1.96$ $\mathrm{SD}$ and $d+1.96 \mathrm{SD}$ ) were calculated. Values obtained by $2 \mathrm{D}$ cine PC-MRI were averaged at all $12-14$ points in a cardiac cycle. To evaluate heartbeat-associated changes in the main PV flow, the pulsatility indices (PI) of maximum velocity, average velocity and flow volume rate were calculated using measurements obtained by $2 \mathrm{D}$ cine PC-MRI according to the following equation: $\mathrm{PI}=\left(\mathrm{V}_{\max }^{\prime}-\mathrm{V}^{\prime}{ }_{\min }\right) / \mathrm{V}_{\text {mean }}^{\prime}\left(\mathrm{V}_{\max }^{\prime}\right.$; maximum parameter in a cardiac cycle, $\mathrm{V}_{\text {min }}^{\prime}$; minimum parameter in that cardiac cycle, $\mathrm{V}^{\prime}$ mean; average parameter at all 12-14 points in that cardiac cycle). Statistical analysis was performed using GraphPad Prism for Windows, version 5.0, 8 (GraphPad Software, La Jolla, CA, USA). Statistical significance was defined as $p<0.05$. The Pearson correlation test was used to assess the correlation between PV flow volume rate in the second-order branches and corresponding liver parenchymal weight.

\section{Results}

\section{Assessment of flow in the main PV by 2D cine PC- MRI and 3D PC-MRI}

Flow in the main PV was analyzed by means of $2 \mathrm{D}$ cine PC-MRI and 3D PC-MRI, as illustrated in Fig. 1a (2D cine PC-MRI) and Fig. 2a (3D PC-MRI). First, magnitude images (Fig. 1b) and velocity images (Fig. 1c) were obtained from five rats using cardiac-gated 2D cine PC-MRI. Flow volume rate (Fig. 1d), maximum flow velocity, measured in the center of the vessel as seen in Fig. 1c, and average flow velocity (Fig. 1e) during a single cardiac cycle were calculated and plotted. Pulsatility indices for maximum and average flow velocities were relatively low, at 0.08 and 0.13 , respectively, demonstrating that PV flow is fairly steady. ${ }^{9}$ Based on this finding, we performed 3D PC-MRI without ECG-synchronization and with time-averaging in the following experiments, to shorten data acquisition time as well as to improve flow data quality. ${ }^{9}$

Second, flow data for the whole PV tree was acquired from the same five rats using 3D PC-MRI. The same site assessed by 2D cine PC-MRI was assessed again using 3D PC-MRI data. Fig. 2a maps out flow velocity, obtained from magnitude images by $3 \mathrm{D}$ PC-MRI, as color-coded streamlines on a PV tree image, obtained from time-of-flight $\mathrm{MR}$ angiography. Average and

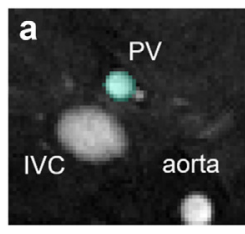

${ }^{b}$ Cardiactrame

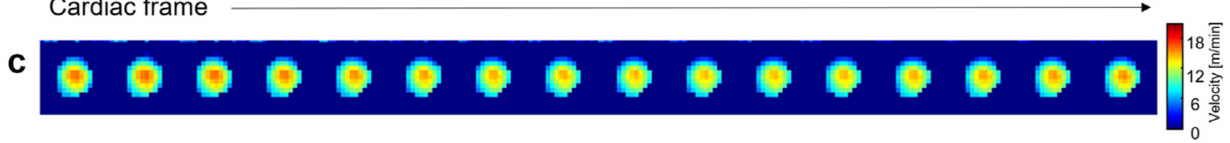

d

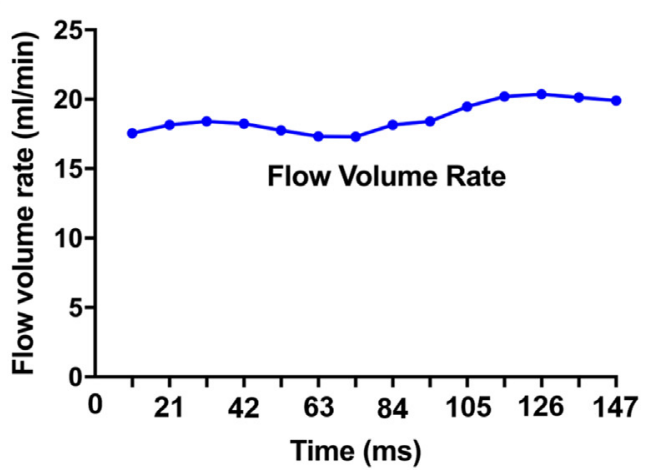

e

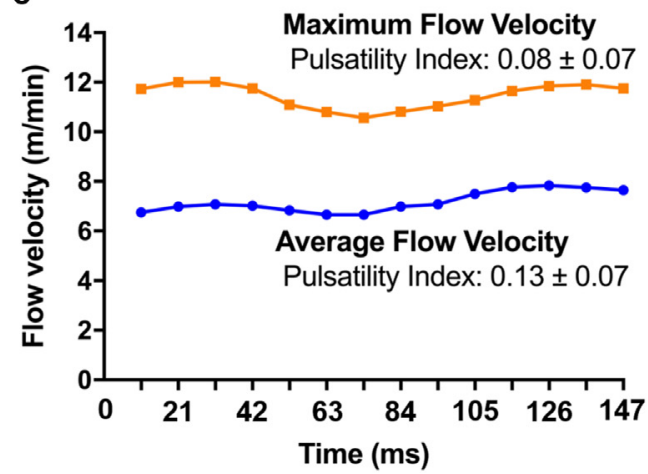

Figure 1 Assessment of the main PV using respiratory- and cardiac-gated 2D cine PC-MRI. An MR angiography image (a) shows the plane of assessment, which includes the PV, IVC, and aorta. Representative magnitude images (b) and velocity images (c) of the main PV are shown as sequential images during one cardiac cycle with a temporal resolution of $10.5 \mathrm{~ms}$. Hemodynamic changes during a single cardiac cycle are shown, revealing flow volume rates (d) and maximum and average flow velocities (e). PV, portal vein; IVC, inferior vena cava; 2D cine PC-MRI, two-dimensional phase-contrast MRI 

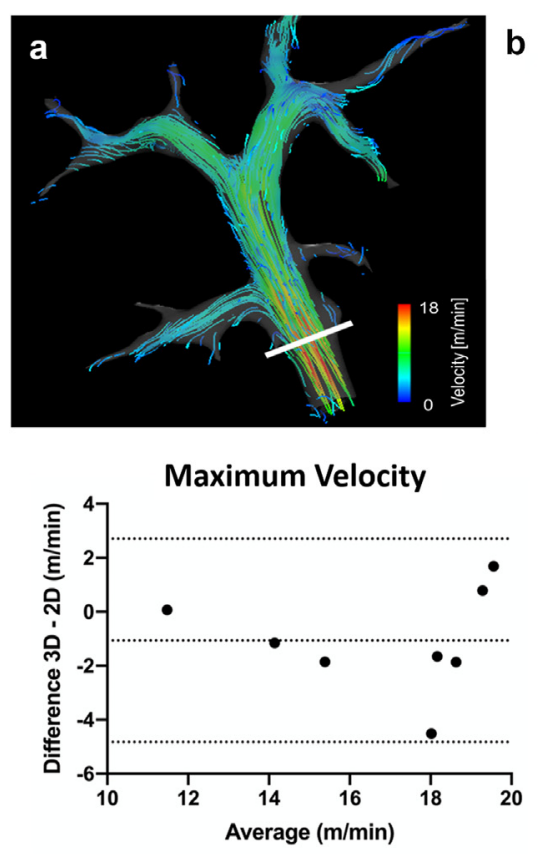

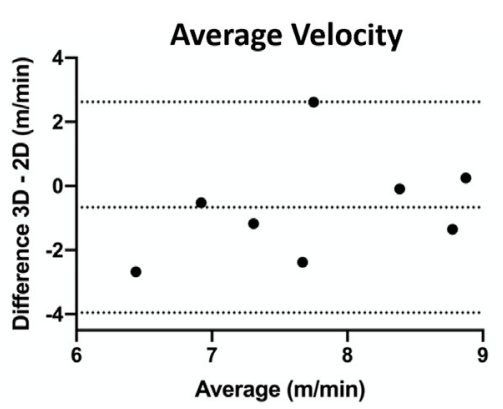

WSS
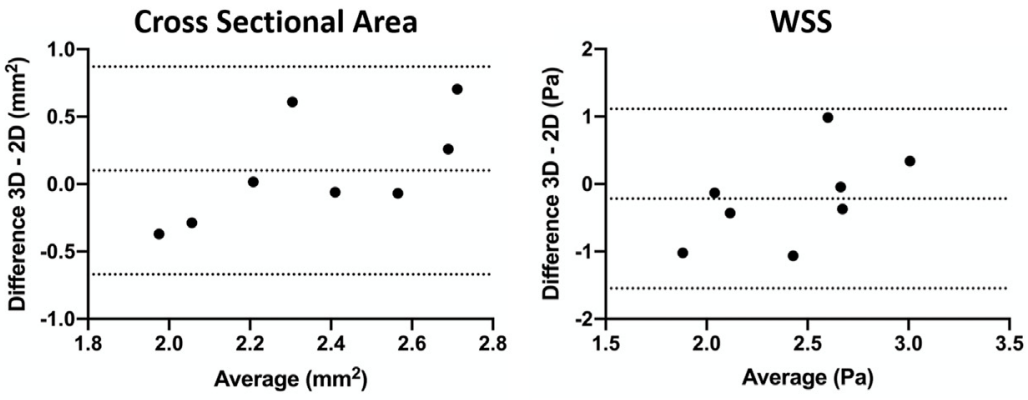

Figure 2 Assessment of the main PV using 3D PC-MRI. (a) The streamlines are visualized as color-coded lines, overlaid on a PV tree image obtained from time-of-flight MR angiography. The plane for flow assessment is indicated by the white line. (b) Bland-Altman plot of data obtained by 2D cine PC-MRI and that obtained by 3D PC-MRI for flow volume rate, average and maximum velocities, cross-sectional area, and WSS. The dashed lines indicate bias, lower and higher limits of agreement. 2D cine PC-MRI, two-dimensional phase-contrast MRI; 3D PC-MRI, three-dimensional phase-contrast MRI; PV, portal vein; WSS, wall shear stress. Each bar represents the IQR

maximum velocities and portal cross-sectional area were recorded and hemodynamic analysis was conducted to quantify flow volume rate and WSS (Table 1). The difference between the measurements obtained by 3D PC-MRI vs. those obtained by $2 \mathrm{D}$ cine PC-MRI was calculated to be $7.9 \%$ for flow volume rate, $10.8 \%$ for average flow velocity, $8.3 \%$ for maximum flow velocity, $0.9 \%$ for cross-sectional area, and $9.6 \%$ for WSS (Table 1 ). The cumulative results of the 3D PC-MRI/2D cine PC-MRI comparisons are summarized in the Bland-Altman plots in Fig. 2b. The five flow parameters of the main PV all demonstrated good agreements between the newly-adopted 3D PCMRI and 2D cine PC-MRI, the previous standard method, demonstrating that there was no consistent bias and the data obtained by 3D PC-MRI was valid and reliable.

\section{Assessment of PV flow in the whole PV tree by 3D PC-MRI}

The flow data for the whole PV tree was acquired by 3D PC-MRI from five rats before and after the meal challenge. Hemodynamic analysis was performed to create $3 \mathrm{D}$ mappings for streamlines and WSS on a PV tree image obtained from time-of-flight MR angiography, showing a complex spatial distribution and subtle flow changes after the meal challenge (Fig. 3a-d). All the intrahepatic PV branches, including the branches to the whole liver (1:main PV), middle and left lateral (2), left median and left lateral (3), right median (4), left median (5), left lateral (6), right (7), and caudate lobes (8), were clearly depicted, and hemodynamic parameters were quantified in eight planes as illustrated in Fig. 3a. The flow volume rate in each plane and the

Table 1 Hemodynamic parameters in the main PV assessed by 2D cine PC-MRI and 3D PC-MR

\begin{tabular}{|c|c|c|c|c|}
\hline & 2D cine PC-MRI & 3D PC-MRI & Difference 3D - 2D (\%) & $P$ value \\
\hline Flow Volume Rate (ml/min) & $18.6 \pm 2.1$ & $17.1 \pm 2.6$ & $-7.9 \%$ & n.s. \\
\hline Average Velocity (m/min) & $8.2 \pm 1.5$ & $7.4 \pm 2.3$ & $-10.8 \%$ & n.s. \\
\hline Maximum Velocity (m/min) & $18.8 \pm 4.3$ & $16.5 \pm 5.4$ & $-8.3 \%$ & n.s. \\
\hline Cross-sectional Area $\left(\mathrm{mm}^{2}\right)$ & $2.3 \pm 0.0$ & $2.5 \pm 0.8$ & $-0.9 \%$ & n.s. \\
\hline Wall Shear Stress (Pa) & $2.5 \pm 0.7$ & $2.2 \pm 1.1$ & $-9.6 \%$ & n.s. \\
\hline
\end{tabular}

2D cine PC-MRI, two-dimensional phase-contrast MRI; 3D PC-MRI, three-dimensional phase-contrast MRI. n.s.; not significant. Data are expressed as the median \pm interquartile range (IQR). 


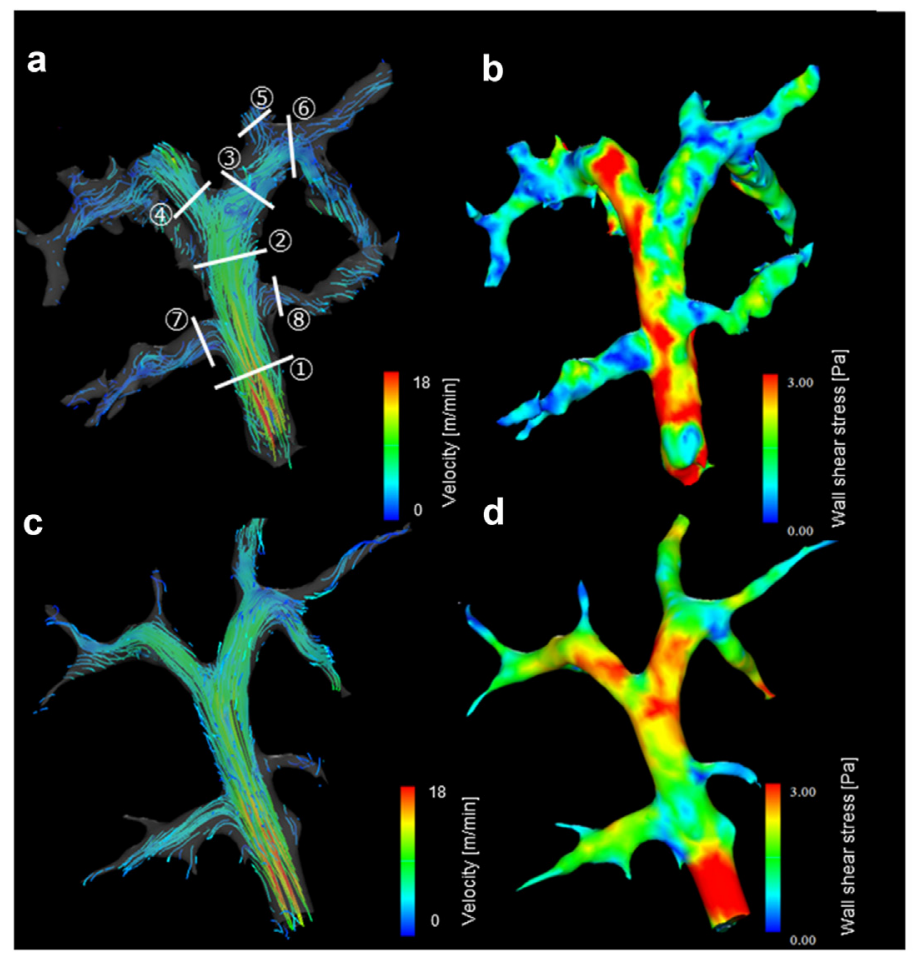

e

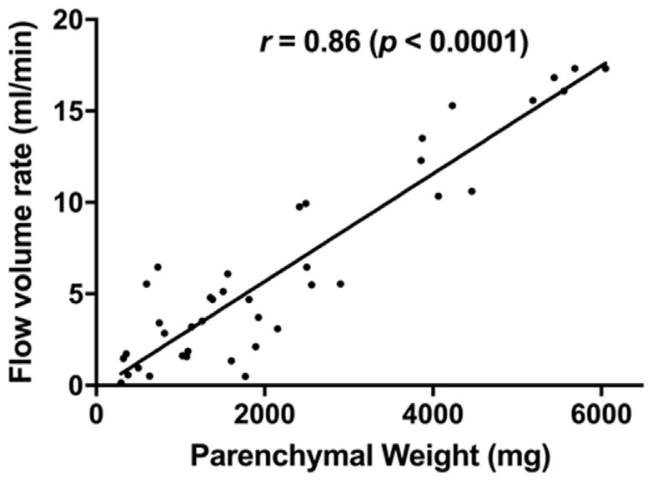

Figure 3 Assessment of PV tributaries by 3D PC-MRI. Representative data of the streamlines (a) and WSS (b) prior to meal intake were mapped out, overlaid on a PV tree image. The planes of assessment are shown: 1, the main PV; 2 , the branch feeding the middle and left lateral; 3 , the branch feeding the left median and left lateral lobes; 4 , the branch to the right median lobe; 5 , the branch to the left median lobe; 6 , the branch to the left lateral lobe; 7 , the branch to the right lobe; 8 , the branch to the caudate lobe. The streamlines (c) and WSS mapping (d) in the same animal after meal intake are shown as an indicator of PV flow changes in response to meal intake. The flow volume rate of each PV branch and the corresponding parenchymal weight are plotted, showing a strong correlation between the two parameters (a correlation coefficient of $0.86, p<0.0001)(e)$

corresponding parenchymal weight are illustrated in Fig. 3e. These values were significantly correlated with a correlation coefficient of $0.86(p<0.0001)$, suggesting that the flow data from the first- and second-order PV branches were valid and precise.

Table 2 summarizes the hemodynamic parameters, i.e., the flow volume rate, average and maximum flow velocities, crosssectional area, and WSS, in the eight planes before and after the meal challenge. In the whole liver, as assessed in the main PV (plane 1), the flow volume rate significantly increased by $13.1 \%$ after meal intake $(p=0.02)$. Cross-sectional area increased by $4.0 \%$, although this difference was not significant (Fig. 4a, c). Maximum flow velocity increased by $9.0 \%$ (not significant), whereas average flow velocity was similar before and after the meal challenge. These data suggest that the increase in flow volume rate in the main PV is attributable to the increase in cross-sectional area. WSS was similar before and after the meal challenge.

As for the first- and second-order PV branches, changes in the flow volume rate in each tributary are illustrated in Fig. $4 a$ and b. Flow volume rate increased after the meal challenge in five of the seven planes, with a significant increase seen in plane $2(p=0.03)$ and plane $7(p=0.01)$; this trend was not seen in the other three lobes (planes 3, 4 and 5). Changes in the other parameters as calculated in four representative branches are illustrated in Fig. 4c. WSS was similar before and after the meal challenge in all branches.

\section{Discussion}

To date, no optimal method of assessing intrahepatic PV flow has been established. This study describes a novel imaging approach in which 3D PC-MRI is combined with hemodynamic analysis, allowing us to acquire flow data for the whole PV tree in rats, including that within the very narrow first- and second-order PV branches. In addition, flow imaging method enables us to measure previously-unmeasurable hemodynamic values such as streamlines, flow volume rate, and WSS. Our findings demonstrate that 3D PC-MRI with hemodynamic analysis is a promising tool for assessing PV flow and that it may lead to a breakthrough in our understanding of PV flow's interaction with liver disease/regeneration in small animal models. 
Table 2 Hemodynamic parameters in the eight planes before and after the meal challenge, and the parenchymal weight corresponding each plane

\begin{tabular}{|c|c|c|c|c|c|c|c|}
\hline & & $\begin{array}{l}\text { Flow volume rate } \\
\text { (mL/min) }\end{array}$ & $\begin{array}{l}\text { Average } \\
\text { velocity } \\
\text { (m/min) }\end{array}$ & $\begin{array}{l}\text { Maximum velocity } \\
\text { (m/min) }\end{array}$ & $\begin{array}{l}\text { Cross- } \\
\text { sectional } \\
\text { area }\left(\mathrm{mm}^{2}\right)\end{array}$ & $\begin{array}{l}\text { Wall shear } \\
\text { stress } \\
\text { (Pa) }\end{array}$ & $\begin{array}{l}\text { Corresponding } \\
\text { parenchymal } \\
\text { weight } \\
\left(10^{2} \times \mathrm{mg}\right)\end{array}$ \\
\hline Plane 1 & $\begin{array}{l}\text { pre/post } \\
\text { meal }\end{array}$ & $\begin{array}{r}16.8 \pm 1.5 / \\
19.0 \pm 1.6\end{array}$ & $\begin{array}{l}6.7 \pm 3.2 / \\
\quad 6.8 \pm 1.6\end{array}$ & $14.5 \pm 6.7 / 15.8 \pm 4.5$ & $\begin{array}{l}2.5 \pm 1.0 / \\
2.6 \pm 0.5\end{array}$ & $\begin{array}{l}2.0 \pm 1.5 / \\
1.9 \pm 0.7\end{array}$ & $55.6 \pm 5.5$ \\
\hline Plane 2 & $\begin{array}{c}\text { pre/post } \\
\text { meal }\end{array}$ & $\begin{array}{l}12.3 \pm 3.9 / 14.5 \pm 2 \\
\quad 0.7\end{array}$ & $\begin{aligned} & 5.6 \pm 1.9 / \\
& 5.6 \pm 1.0\end{aligned}$ & $12.0 \pm 3.5 / 11.4 \pm 3.5$ & $\begin{array}{l}2.0 \pm 0.5 / \\
2.5 \pm 0.9\end{array}$ & $\begin{array}{l}1.8 \pm 1.7 / \\
1.7 \pm 0.5\end{array}$ & $40.7 \pm 4.8$ \\
\hline Plane 3 & $\begin{array}{c}\text { pre/post } \\
\text { meal }\end{array}$ & $6.5 \pm 4.3 / 7.7 \pm 3.2$ & $\begin{aligned} 3.2 & \pm 1.5 / \\
& 4.0 \pm 1.6\end{aligned}$ & $7.4 \pm 2.6 / 8.3 \pm 2.1$ & $\begin{array}{l}1.9 \pm 0.6 / \\
1.9 \pm 0.6\end{array}$ & $\begin{array}{l}1.1 \pm 0.5 / \\
1.5 \pm 0.6\end{array}$ & $25.0 \pm 2.8$ \\
\hline Plane 4 & $\begin{array}{c}\text { pre/post } \\
\text { meal }\end{array}$ & $4.8 \pm 0.9 / 5.7 \pm 1.8$ & $\begin{array}{l}3.5 \pm 0.9 / \\
3.3 \pm 1.3\end{array}$ & $6.8 \pm 2.0 / 6.6 \pm 1.5$ & $\begin{array}{l}1.6 \pm 0.4 / \\
1.6 \pm 0.3\end{array}$ & $\begin{array}{l}1.5 \pm 0.4 / \\
1.2 \pm 0.6\end{array}$ & $15.1 \pm 3.2$ \\
\hline Plane 5 & $\begin{array}{c}\text { pre/post } \\
\text { meal }\end{array}$ & $3.4 \pm 4.3 / 5.7 \pm 4.8$ & $\begin{array}{l}1.8 \pm 1.2 / \\
2.4 \pm 1.1\end{array}$ & $4.7 \pm 2.5 / 5.5 \pm 4.5$ & $\begin{array}{l}1.6 \pm 2.1 / \\
2.0 \pm 1.3\end{array}$ & $\begin{array}{l}0.5 \pm 0.6 / \\
0.9 \pm 0.5\end{array}$ & $7.3 \pm 1.6$ \\
\hline Plane 6 & $\begin{array}{c}\text { pre/post } \\
\text { meal }\end{array}$ & $2.1 \pm 2.5 / 1.8 \pm 4.5$ & $\begin{array}{l}1.5 \pm 1.2 / \\
1.7 \pm 0.7\end{array}$ & $3.5 \pm 2.7 / 3.1 \pm 2.2$ & $\begin{aligned} 2.0 & \pm 1.6 / \\
1.2 & \pm 1.8\end{aligned}$ & $\begin{array}{l}0.4 \pm 0.5 / \\
0.7 \pm 0.3\end{array}$ & $18.9 \pm 3.5$ \\
\hline Plane 7 & $\begin{array}{l}\text { pre/post } \\
\text { meal }\end{array}$ & $1.9 \pm 1.8 / 4.2 \pm 0.5$ & $\begin{array}{l}1.6 \pm 3.2 / \\
3.2 \pm 0.7\end{array}$ & $3.1 \pm 2.0 / 5.3 \pm 1.0$ & $\begin{array}{c}1.0 \pm 0.6 / \\
1.4 \pm 0.2\end{array}$ & $\begin{array}{l}0.8 \pm 1.2 / \\
1.3 \pm 0.4\end{array}$ & $10.9 \pm 1.5$ \\
\hline Plane 8 & $\begin{array}{l}\text { pre/post } \\
\text { meal }\end{array}$ & $1.0 \pm 1.2 / 0.6 \pm 0.6$ & $\begin{aligned} & 1.4 \pm 1.1 / \\
& 0.7 \pm 0.6\end{aligned}$ & $3.1 \pm 1.1 / 2.5 \pm 2.0$ & $\begin{array}{l}0.8 \pm 0.3 / \\
0.7 \pm 0.4\end{array}$ & $\begin{array}{l}0.8 \pm 0.5 / \\
0.4 \pm 0.3\end{array}$ & $3.6 \pm 1.3$ \\
\hline
\end{tabular}

Several modalities have been used to evaluate PV flow in small animals and humans. In humans, Doppler ultrasound has been the standard modality for this purpose; ${ }^{10}$ yet it often yields incomplete or inaccurate hemodynamic data due to the limited size of acoustic windows, which can be compounded by overlying gas-filled bowels, ${ }^{11}$ observer variability, ${ }^{12}$ the difficulty of visualizing complex PV branches, ${ }^{11}$ and the inability to measure three-dimensionally-variable flow using a $2 \mathrm{D}$ scanning a

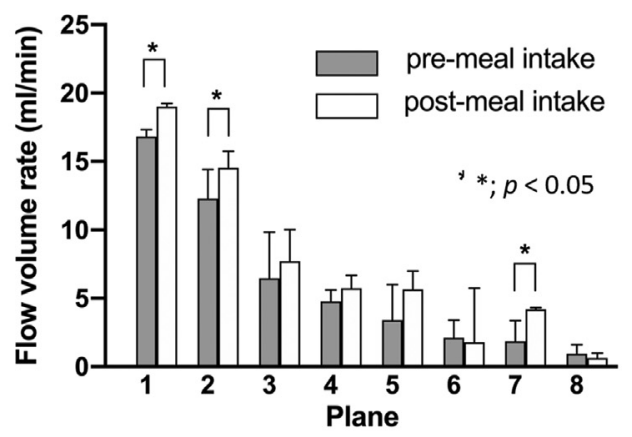

C

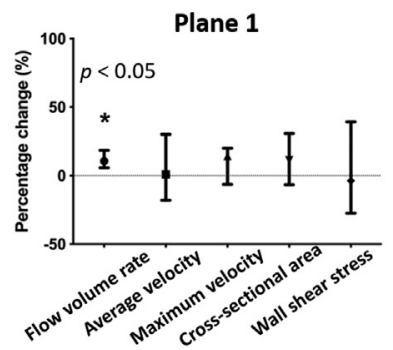

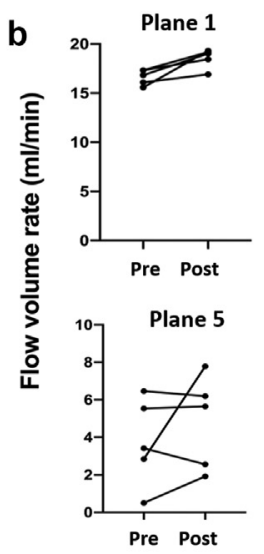
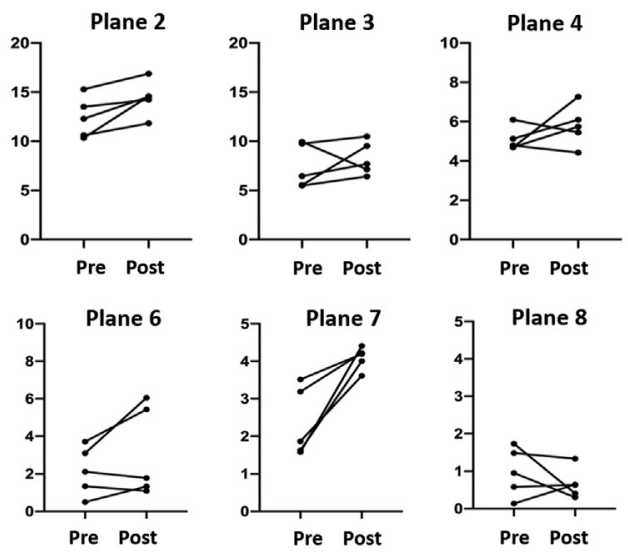

Plane 3
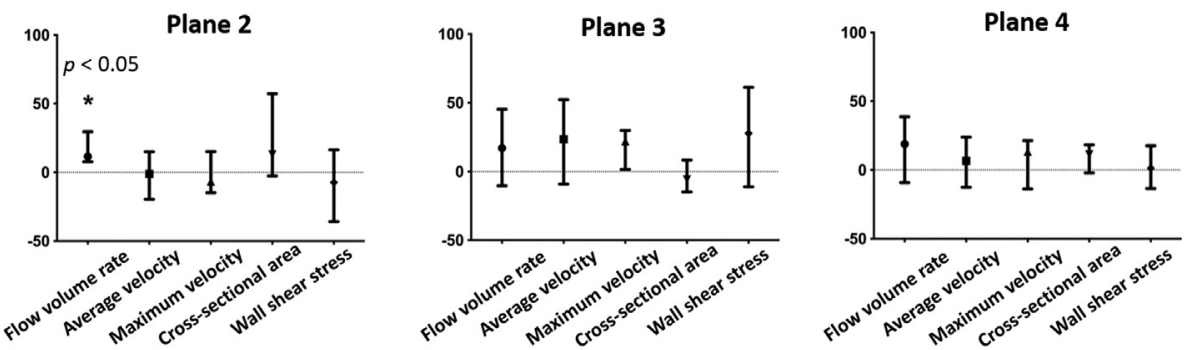

Figure 4 Changes in flow volume rate in response to a meal challenge for all eight analysis planes. (a) $(n=5)$ Each bar represents the IQR. Flow volume rate increased significantly in planes $1(p=0.02), 2(p=0.03)$ and $7(p=0.01)$. The graphs represent the changes in flow volume rate in all eight planes in all five rats (b). Percentage changes for all measurements in four planes $(c) .{ }^{*}=$ significant difference compared to data taken before the meal challenge, $p<0.05)$ 
technique. In small animals, flow meters, ${ }^{13-15}$ lase specs, ${ }^{16}$ and microspheres, ${ }^{17,18}$ have been used to evaluate PV flow, but these modalities are too invasive to be used repeatedly, and the flow velocity data they provide is insufficiently detailed.

Alternatively, several studies have used MRI to evaluate PV flow in humans and rodents, ${ }^{19,20}$ concluding that MRI offers several advantages over the aforementioned modalities, including noninvasiveness, objectivity as opposed to examiner dependence, and a three-dimensional scanning area. Yet even 2D cine PC-MRI, the standard MRI method to date, only detects flow velocity perpendicular to a single cross-sectional scanning plane, and so does not enable flow assessment of the entire PV tree. In this way, 3D MRI offers an advantage over 2D cine PC-MRI in that it captures data related to all flow vectors in all directions over the whole scanning area, $^{21,22}$ which enables retrospective flow analyses in any PV branch of interest using the acquired data. Because this technology has not frequently been applied to the liver or to PV flow assessment, this study aimed to confirm its usefulness by comparing a dataset obtained by 3D PC-MRI with one obtained in the same area by $2 \mathrm{D}$ PC-MRI as well as with previously reported data on the rat PV obtained by 2D PC-MRI. ${ }^{20}$ Average flow velocity obtained by 3D PC-MRI, by 2D PC-MRI, and from the reported data, ${ }^{20}$ was $7.4 \pm 2.3,8.2 \pm 1.5$, and $7.0 \pm 1.7 \mathrm{~m} / \mathrm{min}$, respectively; flow volume rate obtained from these three sources was $17.1 \pm 2.6,18.6 \pm 2.1$, and $20 \pm 7 \mathrm{~mL} / \mathrm{min}$, respectively.

An increase in PV flow after oral intake has been demonstrated in humans, ${ }^{23-25}$ but, to date, we have lacked the ability to quantify this increase with precision in small animals. ${ }^{26}$ This study detected small flow changes in the narrow PV branches after a meal challenge, indicating that, for the first time, small flow changes in the narrow intrahepatic PV branches of small animals are available for study. PV flow dynamics can now be accurately and serially monitored with minimal harm to animals, and complex flow characteristics can be quantified in terms of previouslyunmeasurable parameters. One research project where this new method will be particularly useful is the study of PV hyperperfusion in advanced cirrhosis and its association with uneven morphologic changes, whereby PV flow changes non-uniformly as the liver stiffens, which may cause right liver atrophy with left and caudate lobe hypertrophy. ${ }^{27}$ Another potential application of our novel method is in examining PV flow changes after surgical interventions such as hepatectomy, liver transplantation, or portal vein embolization, where increased WSS or mechanical stress to the PV endothelium may trigger liver regeneration after an intervention. ${ }^{28}$ It is a critical goal in practice to identify an optimal range of PV flow that will induce normal liver regeneration and to elucidate the mechanisms that sometimes limit liver recovery, as in the so-called small-for-size syndrome, which may be linked to excessive hemodynamic stress. ${ }^{28}$

This approach has several limitations inherent to its use of MRI. First, the anesthesia, which is unavoidable in an MRI study using rats, could have added more variability and uncertainty to the measurements. The relatively long duration associated with data acquisition in MRI compared with other modalities such as CT scan or US may limit the use of our method. The application of $3 \mathrm{D}$ cine PC-MRI, which currently requires much longer data acquisition time compared with 3D non-cine PC MRI, and analyses of pulsatility changes in the PV flow would be a future task. Although little has been reported on abdominal MRI using 7TMRI, its high expense compared with 3T-MRI and potential health hazards associated with its superhigh magnetic field could be hurdles to disseminate the approach, when expanding this technology to human.

\section{Conclusion}

Our imaging method combining 3D PC-MRI at 7T and hemodynamic analysis is a promising tool for noninvasive serial assessment of intrahepatic portal vein flow. It enables the measurement of previously-unmeasurable hemodynamic parameters in very narrow branches, which will allow future studies to investigate portal vein hemodynamics associated with liver disease/postoperative liver recovery in small animals in detail.

\section{Acknowledgments}

The MRI data presented in this work were obtained in the Division for Small Animal MRI, Medical Research Support Center, Graduate School of Medicine, Kyoto University, Japan.

\section{Author contributions}

Study concept/design: YO, SO, MN.

Data acquisition: YO, HI, SW, KT and T Ito.

Data analysis/interpretation: YO, SO, MN, KF, T Ishii, TM, KT.

Drafting of the manuscript: YO, and SO.

Critical revision of the manuscript: HI, MN, SW, KT, T Ito, KF, T Ishii, TM, KT.

Final approval of the manuscript: YO, SO, HI, MN, SW, KT, T Ito, KF, T Ishii, TM, KT.

\section{Funding}

The research was supported by Japan Society for the Promotion of Science (Grant Number 19K18112).

\section{Conflict of interest}

None declared.

\section{References}

1. Lorenz L, Axnick J, Buschmann T, Henning C, Urner S, Fang S et al. (2018) Mechanosensing by $\beta 1$ integrin induces angiocrine signals for liver growth and survival. Nature 562:128-132.

2. Dahm F, Georgiev P, Clavien PA. (2005) Small-for-size syndrome after partial liver transplantation: definition, mechanisms of disease and clinical implications. Am J Transplant 5:2605-2610.

3. Ogura Y, Hori T, El Moghazy WM, Yoshizawa A, Oike F, Mori A et al. (2010) Portal pressure $<15 \mathrm{~mm} \mathrm{Hg}$ is a key for successful adult living donor liver transplantation utilizing smaller grafts than before. Liver Transpl 16:718-728. 
4. Yao S, Kaido T, Uozumi R, Yagi S, Miyachi Y, Fukumitsu K et al. (2018) Is portal venous pressure modulation still indicated for all recipients in living donor liver transplantation? Liver Transp/ 24:1578-1588.

5. Stankovic Z, Frydrychowicz A, Csatari Z, Panther E, Deibert P, Euringer W et al. (2010) MR-based visualization and quantification of three-dimensional flow characteristics in the portal venous system. $J$ Magn Reson Imaging 32:466-475.

6. Frydrychowicz A, Landgraf BR, Niespodzany E, Verma RW, RoldánAlzate A, Johnson KM et al. (2011) Four-dimensional velocity mapping of the hepatic and splanchnic vasculature with radial sampling at 3 tesla: a feasibility study in portal hypertension. J Magn Reson Imaging 34:577-584.

7. Ogiso S, Nakamura M, Tanaka T, Komiya K, Kamei H, Onishi Y et al. (2020) Computational fluid dynamics-based blood flow assessment facilitates optimal management of portal vein stenosis after liver transplantation. J Gastrointest Surg 24:460-461.

8. Peng SL, Shih CT, Huang CW, Chiu SC, Shen WC. (2017) Optimized analysis of blood flow and wall shear stress in the common carotid artery of rat model by phase-contrast MRI. Sci Rep 7:5253.

9. Landgraf BR, Johnson KM, Roldán-Alzate A, Francois CJ, Wieben O, Reeder SB. (2014) Effect of temporal resolution on 4D flow MRI in the portal circulation. J Magn Reson Imaging 39:819-826.

10. Görg C, Riera-Knorrenschild J, Dietrich J. (2002) Pictorial review: colour Doppler ultrasound flow patterns in the portal venous system. $\mathrm{Br} J$ Radiol 75:919-929.

11. Finn JP, Kane RA, Edelman RR, Jenkins RL, Lewis WD, Muller M et al. (1993) Imaging of the portal venous system in patients with cirrhosis: MR angiography vs duplex Doppler sonography. AJR Am J Roentgenol 161:989-994.

12. de Vries PJ, van Hattum J, Hoekstra JB, de Hooge P. (1991) Duplex Doppler measurements of portal venous flow in normal subjects. Interand intra-observer variability. J Hepatol 13:358-363.

13. Dili A, Bertrand C, Lebrun V, Pirlot B, Leclercq IA. (2019) Hypoxia protects the liver from small for size syndrome: a lesson learned from the associated liver partition and portal vein ligation for staged hepatectomy (ALPPS) procedure in rats. Am J Transplant 19:2979-2990.

14. Hsin IF, Huang HC, Chang CC, Hsu SJ, Lee FY, Huo TI et al. (2018) Insulin reverses major portal hypertension-related derangements in rats with liver cirrhosis and diabetes. Clin Sci (Lond) 132:2391-2405.

15. Yoshida D, Akahoshi T, Kawanaka H, Yamaguchi S, Kinjo N, Taketomi A et al. (2011) Roles of vascular endothelial growth factor and endothelial nitric oxide synthase during revascularization and regeneration after partial hepatectomy in a rat model. Surg Today 41:1622-1629.
16. Jin WX, Wang $B$, Zhang $Y L$, Dong R, Wang XB, Guo JM et al. (2018) Effects of hepatic blood inflow on liver ultrastructure and regeneration after extensive liver resection in rats with cirrhosis. Exp Ther Med 16: 2573-2583.

17. Um SH, Nishida O, Tokubayashi M, Kimura F, Takimoto $Y$, Yoshioka H et al. (1994) Hemodynamic changes after ligation of a major branch of the portal vein in rats: comparison with rats with portal vein constriction. Hepatology 19:202-209.

18. Van de Casteele M, Sägesser H, Zimmermann H, Reichen J. (2001) Characterisation of portal hypertension models by microspheres in anaesthetised rats: a comparison of liver flow. Pharmacol Ther 90:35-43.

19. Lee H, Lee J, Joe E, Yang S, Song JE, Choi YS et al. (2017) Flowsuppressed hyperpolarized. Magn Reson Med 78:1674-1682.

20. Schaffner $D$, von Elverfeldt $D$, Deibert $P$, Lazaro $A$, Merfort I, Lutz L et al. (2017) Phase-contrast MR flow imaging: A tool to determine hepatic hemodynamics in rats with a healthy, fibrotic, or cirrhotic liver. J Magn Reson Imaging 46:1526-1534.

21. Markl M, Frydrychowicz A, Kozerke S, Hope M, Wieben O. (2012) 4D flow MRI. J Magn Reson Imaging 36:1015-1036.

22. Yamashita $S$, Isoda $H$, Hirano $M$, Takeda $H$, Inagawa $S$, Takehara $Y$ et al. (2007) Visualization of hemodynamics in intracranial arteries using time-resolved three-dimensional phase-contrast MRI. J Magn Reson Imaging 25:473-478.

23. Pazahr S, Nanz D, Rossi C, Chuck N, Stenger I, Wurnig MC et al. (2014) Magnetic resonance imaging of the liver: apparent diffusion coefficients from multiexponential analysis of b values greater than $50 \mathrm{~s} / \mathrm{mm}^{2}$ do not respond to caloric intake despite increased portal-venous blood flow. Invest Radiol 49:138-146.

24. Schalkx HJ, Petersen ET, Peters NH, Veldhuis WB, van Leeuwen MS, Pluim JP et al. (2015) Arterial and portal venous liver perfusion using selective spin labelling MRI. Eur Radiol 25:1529-1540.

25. Roldán-Alzate A, Frydrychowicz A, Said A, Johnson KM, Francois CJ, Wieben $O$ et al. (2015) Impaired regulation of portal venous flow in response to a meal challenge as quantified by 4D flow MRI. $J$ Magn Reson Imaging 42:1009-1017.

26. Rani SD, Nemanich ST, Fettig N, Shoghi KI. (2013) Kinetic analysis of FDG in rat liver: effect of dietary intervention on arterial and portal vein input. Nucl Med Biol 40:537-546.

27. Brancatelli G, Federle MP, Ambrosini R, Lagalla R, Carriero A, Midiri M et al. (2007) Cirrhosis: CT and MR imaging evaluation. Eur J Radiol 61: 57-69.

28. Rabbany SY, Rafii S. (2018) Blood flow forces liver growth. Nature 562: $42-43$. 